\title{
Original
}

\section{Relationship of Grade of Malignant Brain Tumor to Cancer Stem Cells and Survivin Expression}

\author{
Yusuke KOBAYASHI ${ }^{1)}$, Akiko SASAKI ${ }^{2)}$, Mayumi TsuJI ${ }^{2)}$, \\ Yuko UdakA $^{2)}$, Hideto OYAmada ${ }^{2)}$, Yuko TsunOdA ${ }^{3)}$, \\ Shinichi IwaI ${ }^{4)}$, Masayuki SomeI ${ }^{2)}$, Junichiro KIZAKI ${ }^{2)}$, \\ Yasuyuki Kondo ${ }^{2)}$, Katsuyoshi SHIMIzu ${ }^{1)}$ and Tohru Mizutani ${ }^{1)}$
}

\begin{abstract}
Glioblastoma (GBM) is difficult to completely cure by surgical treatment alone, and it is generally treated with a combination of surgery, radiotherapy, and chemotherapy. However, GBM is resistant to radiotherapy and chemotherapy, and complete cure cannot be achieved. Cancer stem cells (CSC) and survivin, which inhibit apoptosis, are considered as factors underlying tumor recurrence and the radiation- and drug-resistance of these tumors. We analyzed CSC and survivin expression in surgically excised specimens of malignant brain tumors to establish the relationships between the grades and CSC and survivin expression and between MIB-1 (Ki-67) expression and resistance. No relationship was noted between the grades and CSC or survivin expression, or between MIB-1 and CSC expression or between Grade 3 and 4 MIB-1 and survivin expression, although a correlation was noted between MIB-1 and survivin expression in Grade II tumors. These findings suggested that CSC are consistently contained in tumor tissue at a specific rate regardless of the histological grade, and the apoptosis of cells with low-level proliferative and cell cycling activities does not occur because these cells do not respond to chemotherapy or radiation, being resistant to treatment.
\end{abstract}

Key words : cancer stem cells, survivin, glioblastoma

\section{Introduction}

Among primary brain tumors, highly malignant, high-grade glioblastoma (59\%) and medulloblastoma (62\%) show a high incidence in males and frequently develop in the 40 50s age group. Glioblastoma (GBM) and medulloblastoma of grade IV, based on the WHO classification, grow rapidly and are fatal if untreated. Histologically, these brain malignancies show abundant cell division and necrosis, rapidly infiltrate the surrounding tissue, and disseminate to the menin $x^{1)}$. GBM and medulloblastoma are therefore highly malignant tumors associated

\footnotetext{
1) Department of Neurosurgery, Showa University School of Medicine, 1-5-8 Hatanodai, Shinagawa-ku, Tokyo 142-8666, Japan.

2) Department of Pharmacology, Showa University School of Medicine, 1-5-8 Hatanodai, Shinagawa-ku, Tokyo 142-8555, Japan.

3) Breast Center, Kameda Medical Center, 929 Higashi-cho, Kamogawa City, Chiba 296-8602, Japan.

4) Department of Healthcare and Regulatory Sciences, Showa University School of Pharmaceutical Sciences, 1-5-8 Hatanodai, Shinagawa-ku, Tokyo 142-8555, Japan.
} 
with a poor prognosis and survival time shorter than 1 year. In addition, the therapeutic outcomes for patients with these tumors have not markedly improved over several decades.

Glioblastoma consists of mature glioma, and reportedly, most are dedifferentiated atypical astrocytoma $^{2)}$ REF. However, undifferentiated pluripotent cells with a self-replicating ability were recently reported in glioblastoma tissue $\mathrm{REF}^{3)}$. Such cells, termed cancer stem cells (CSC), were also identified in acute myelogenous leukemia ${ }^{4)}$, breast cancer ${ }^{5)}$, and other brain tumors $^{6-9)}$, and CSC-targeting treatment methods are now being investigated. CSC are in the G0 phase of the cell cycle, present in tissue as a stem cell-specific side population (SP cells) in various cancer tissues and cancer cell lines including GBM, and have tumor-forming and treatment-resistant properties ${ }^{7,10)}$. Recent investigations have thus focused on developing new anticancer drugs effective for CSC, a radiosensitizer application method aiming at increasing the effect of radiotherapy on CSC, and a CSC-targeting treatment using immunocytes ${ }^{11,12)}$. Tumor progression in glioblastoma was also effectively inhibited by an anti-angiogenic treatment that blocks the vascular niche or portal for CSC and the surrounding tumor cells. Those studies also correlated both CSC expression and survival time with the clinical outcomes of patients with oligodendrocyte tumors, revealing CSC to be a useful prognostic factor ${ }^{13,14)}$.

Survivin belongs to the inhibitors of the apoptosis (IAP) family, which all inhibit apoptosis by inhibiting caspase ${ }^{15)}$. It is strongly expressed in embryonic cells, but it is expressed only in limited organs in adults, including the testis and thymus. Survivin is undetectable in adult tissue, but its overexpression was recently reported in human malignant tumors of the large intestine, breast, stomach, esophagus, liver, and ovary ${ }^{16)}$. Patients overexpressing the survivin gene in their cancer cells have a poor prognosis, and anti-survivin antibody production occurs in the blood of cancer patients $\mathrm{REF}^{17,18)}$. Furthermore, antisense inhibition of survivin gene expression in cancer cells induced apoptosis, and together, these findings prompted investigation of survivin as an immunotherapy target ${ }^{19)}$. However, there is no reported association with either the growth of malignant brain tumors or the WHO grade with CSC and survivin expression pattern. In this study, we analyzed the association between WHO grade and CSC survivin expression to investigate the important relationship between WHO grade and treatment resistance.

\section{Material and Methods}

\section{Subjects}

In this study we investigated specimens surgically excised from 70 patients with malignant brain tumor from 1987 to 2000. The group comprised 32 patients with grade 2 astrocytoma, 4 patients with grade 3 astrocytoma, and 34 patients with grade 4 glioblastoma (Table 1).

\section{Statistical analysis}

Data were tested for significance using ANOVA and Bonferroni. Results were considered significant with a $P$ value $<0.05$. 


\section{Fluorescent immunohistochemistry}

Eighty-two sections in total from the specimens were deparaffinized, and then incubated in $0.3 \% \mathrm{H}_{2} \mathrm{O}_{2}$ for $5 \mathrm{~min}$ to remove endogenous peroxidase. The sections were then incubated in non-specific blocking reagent (X0909, Dako, Glostrup, DK) for $5 \mathrm{~min}$ to block nonspecific staining. Sections were first incubated with anti-PROM1 primary antibody (1:100, Abnova, Taipei, Taiwan) for 1 hour at room temperature, and a secondary, TRIC-conjugated anti-rabbit IgG (A21428, Life Technologies, USA) in a humid chamber at $37^{\circ} \mathrm{C}$ for $30 \mathrm{~min}$. Sequentially, sections were incubated with anti-survivin (1:100, M2624, Dako) and anti- Ki-67 (MIB-1) (1:100, M0722, Dako) primary antibodies at $37^{\circ} \mathrm{C}$ for 1 hour, and then a secondary antibody FITC-conjugated anti-mouse $\operatorname{lgG}$ (A11001, Life Technologies, USA) for $30 \mathrm{~min}$. Sections were systematically counterstained with bisbenzimide H33342 (Hoechst 33342), and images were captured on a confocal microscope (Meta Xpress Image Analysis).

\section{Results}

\section{Clinicopathological characteristics of the patients}

Table 1 lists the clinicopathological characteristics of malignant brain tumors in the 70 patients. To confirm the relationship between malignancy grade and proliferative ability, immunohistochemistry (IHC) analysis was performed using fluorescence labeled antibody detection. Typical morphological characteristics of the malignant brain tumors were examined by HE staining (Fig. 1A, B, C).

\section{Results of immunostaining}

MIB-1 protein is used as an index of prognosis in patients with malignant tumors because it marks cells undergoing nuclear division. The MIB-1-immunopositive rate was $3.72 \%$ in Grade II astrocytoma, 4.57\% in Grade III anaplastic astrocytoma, and 8.8\% in Grade IV glioblastoma, thus the proliferative ability of the cells increased with the histological grade (Fig. 2A).

To detect undifferentiated CSC, we employed IHC using the anti-PROM antibody,

Table 1. Patient backgrounds and gene expressions

\begin{tabular}{lccc}
\hline Histological grade (WHO) & Grade II & Grade III & Grade IV \\
\hline Patients & 32 & 4 & 34 \\
Sex (male / female) & $11 / 21$ & $4 / 0$ & $14 / 20$ \\
Age, medium (range) & 40 & 39.5 & 54.5 \\
MIB-1-positive (\%) & $(9-73)$ & $(30-50)$ & $(11-71)$ \\
PROM-positive (\%) & 3.72 & 4.57 & 8.8 \\
Survivin-positive (\%) & 0.94 & 0.58 & 0.89 \\
\hline
\end{tabular}

All values represent mean $\pm \mathrm{SD}$ (range)

WHO, World Health Organization 


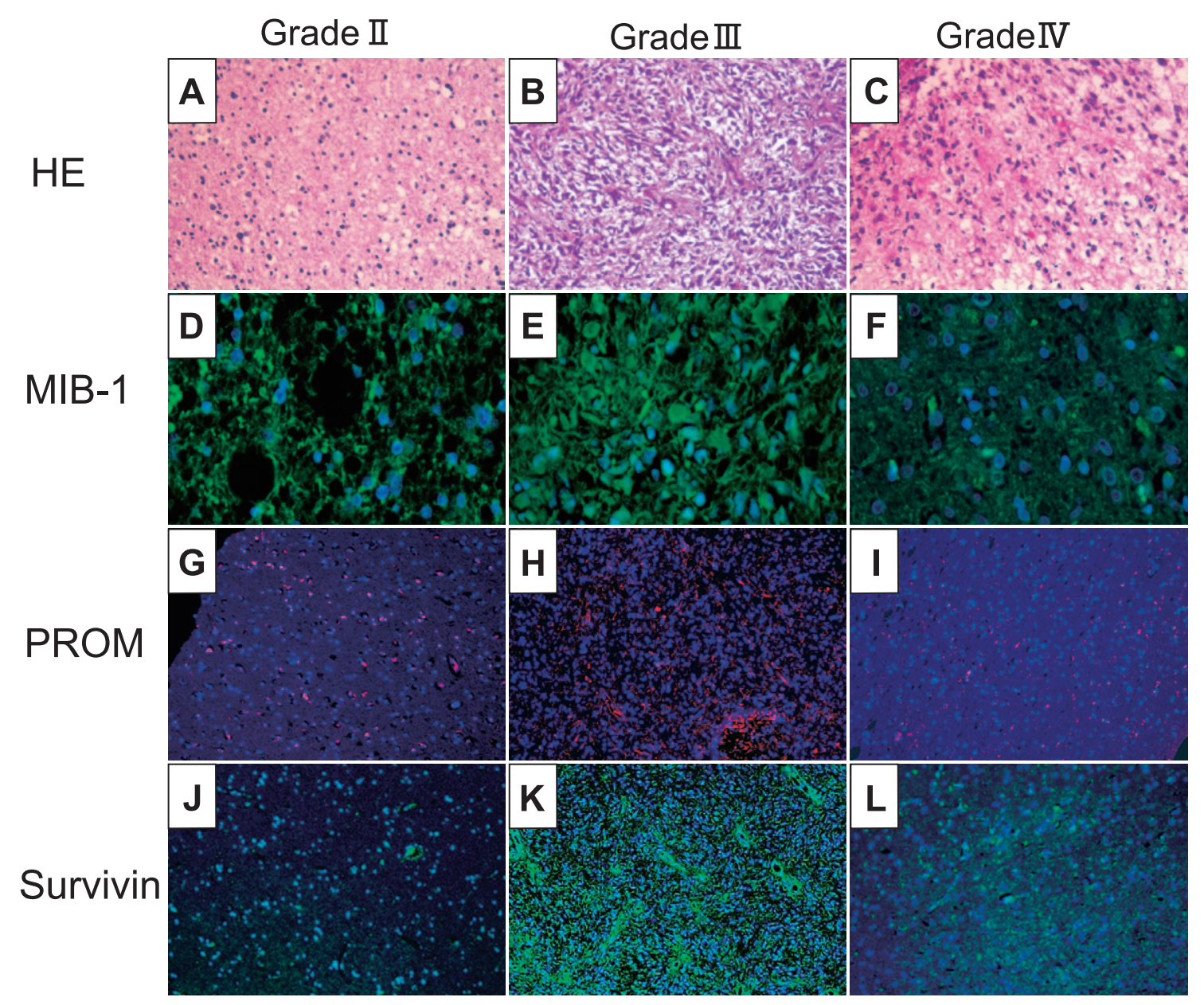

Fig. 1. MIB-1, PROM, and survivin expression in human glioma.

(A-C) Hematoxylin / eosin (HE) -stained sections of Grade II (A), Grade III (B), and Grade IV malignancies (C). MIB-1 expression was detected by immunohistochemistry in Grade II (D), Grade III (E), and Grade IV malignancies (F). PROM expression was detected in Grade II (G), Grade III $(\mathrm{H})$, and Grade IV malignancies (I). Survivin expression was detected in Grade II (J), Grade III (K), and Grade IV malignancies (L).

Magnification $\times 200$.

and confirmed the localization of CSC in the tumor core (Fig. 1G, H, I). The PROMimmunopositive rate was $0.94 \%$ in Grade II astrocytoma, $0.58 \%$ in Grade III anaplastic astrocytoma, and $0.89 \%$ in Grade IV glioblastoma, showing no significant association between undifferentiated CSC rate and grade (Fig. 2B).

We then localized survivin to the tumor cell cytoplasm (Fig. 1J, K, L). The survivinimmunopositive rate was $2.00 \%$ in Grade II astrocytoma, $0.59 \%$ in Grade III anaplastic astrocytoma, and $1.99 \%$ in Grade IV glioblastoma, again showing no significant difference in surviving expression among the tumor grades (Fig. 2C).

Comparison of proliferative ability with PROM and survivin in each grade

PROM and survivin expressions relative to MIB-1 (proliferative ability index) were analyzed 
A

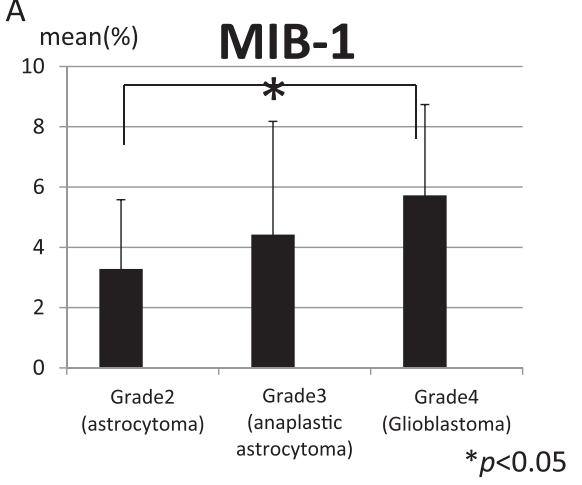

B

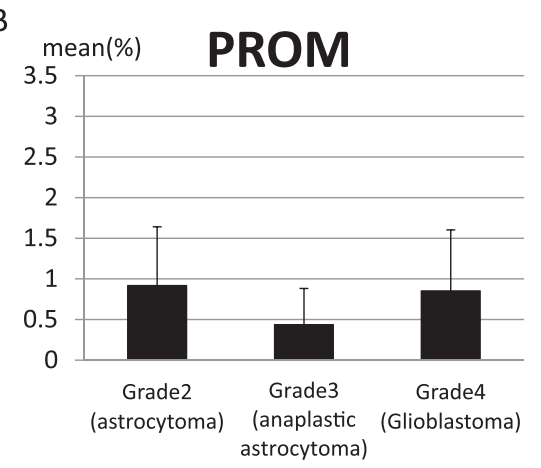

C

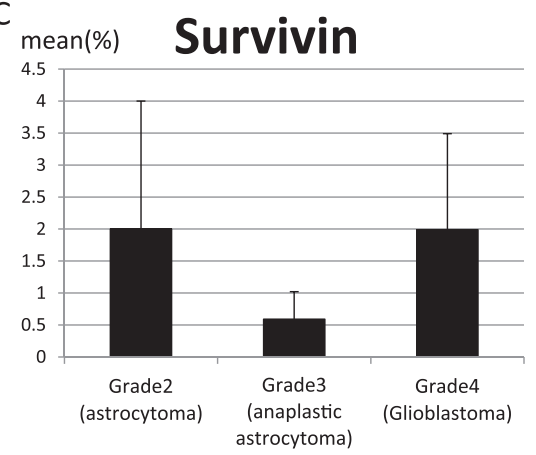

Fig. 2. Significance of differences in MIB-1, PROM, and survivin expression among tumor grades.

(A) A significant difference was noted in MIB-1 expression between Grades 2 and 4 malignancies, but no significant differences were noted in (B) PROM or (C) survivin expression (ANOVA and Bonferroni test).

Table 2. Immunohistochemical analysis of MIB-1 and survivin expression in glioma

\begin{tabular}{cccc}
\hline $\begin{array}{c}\text { Histological grade } \\
\text { (WHO) }\end{array}$ & MIB-1 (\%) & Survivin & $P$ value \\
\hline II & 3.72 & 2.00 & 0.001 \\
III & 4.57 & 0.59 & 0.21 \\
IV & 8.8 & 1.99 & 0.21 \\
\hline
\end{tabular}

Survivin expression of the MIB-1 was significantly different among the tumor grades $(P<0.001)$.

in each grade. No significant correlation was noted between Grade II MIB-1 and Grade II PROM expression, between Grade III MIB-1 and Grade III PROM expression, or between Grade IV MIB-1 and Grade IV PROM expression. However, there was a significant correlation between Grade II MIB-1 and Grade II survivin expression, but not between Grade III MIB-1 and Grade III survivin expression or between Grade IV MIB-1 and Grade IV survivin expression 
(Table 2).

\section{Discussion}

Malignant brain tumors show strong resistance to postoperative chemotherapy and radiotherapy, and a correlation between CSC numbers in the tumor and the resistance mechanism has been suggested as a cause. CSC-induced resistance is considered to derive from the cells' self-replicating and proliferative abilities and drug-eliminating ATP binding cassette (ABC) transporters. Survivin belongs to the inhibitor of apoptosis (IAP) family, and mediates cellular resistance by inhibiting cell death.

The present study found no significant difference in MIB-1expression between Grade II and III malignant brain tumors, but a significantly higher immunopositivity for this protein was noted in Grade IV tumors. However, no significant difference was noted in PROM expression among Grades II, III, and IV. The relationship between MIB-1 and PROM was analyzed in each grade, but no significance was detected, showing that CSC accounted for a constant ratio of cells regardless of the grade of malignant brain tumor. No significant correlation was noted between Grade II MIB-1 and Grade II PROM expression, between Grade III MIB-1 and Grade III PROM expression, or between Grade IV MIB-1 and Grade IV PROM expression. Tumor cells with a high proliferation rate showing active cell cycling and CSC constantly arrested in the G0 phase may be not related to the grading, which is based solely on cells in the growth phase, and thus CSC and resistance due to survivin are not taken into consideration. This study suggested that treatment-resistant CSC in the G0 phase are constantly present at a specific rate in preoperative malignant brain tumors before radiotherapy and chemotherapy. CSC are maintained in the G0 phase (resting phase), with the number, division, and resting phase of CSC controlled through interactions with the environment (tissue microenvironment and cellular niche) around CSC. The microenvironment called a niche for CSC to carry out the work as a stem cell is required. In addition, CSC have an inherent self-replicating ability, and we previously reported that CSC in the G0 phase differentiated and proliferated when cultured malignant brain tumor cells were irradiated at a high $\operatorname{dose}^{20,21)}$, suggesting that, when radiation and chemotherapy are applied as postoperative treatment, it is important to set the treatment target at CSC and reduce the treatment resistance of CSC in the malignant brain tumor by destroying the CSC growthpromoting niche (micro) environment, which in turn induces the differentiation of CSC in the G0 phase, and inhibits molecules necessary for self-replication.

Survivin is an IAP family protein, all of which contain the anti-apoptosis action-determining baculovirus IAP repeat (BIR) domain ${ }^{22)}$. Two splice variants (survivin2B and $-\Delta \mathrm{Ex} 3$ ) are transcribed from the survivin gene, and both have been reported to have an anti-apoptosis function. In addition, survivin expression level is related to the MIB-1 index and has been proposed as an index of tumor cell growth activity ${ }^{23)}$. Regarding apoptosis signals, p53 activated by DNA injury promotes the transcription of Bax and PUMA (p53 up-regulated modulator of apoptosis), which control cytochrome c release by regulating mitochondrial membrane permeability. Cytochrome $\mathrm{c}$ binds to Apaf-1 and forms an active complex with caspase 9, and 
this complex activates caspase 3 and 7, executing apoptosis. Survivin inhibits caspase 9 activity by binding to cofactor XIAP and thus acting as an anti-apoptosis factor. It was recently reported that sensitivity to existing drugs was enhanced by the functional inhibition of survivin using siRNA and ribozyme ${ }^{24,25)}$. In this study, there was no significant difference in survivin expression among Grades II, III, and IV tissues. However, analysis within each grade uncovered a significant correlation between MIB-1- and survivin expression in Grade II tumors. These findings suggested that malignant brain tumors show a specific level of apoptosis resistance regardless of the histological grade, and the proliferative ability and resistance of tumor cells are associated only in low-malignant Grade II brain tumors. Apoptosis resistance in tumor cells with a low proliferative ability could reflect the numerous cells noted in the G0 phase. Since these cells are not actively progressing through the cell cycle, they do not respond to chemotherapy or show radiation sensitivity, and so do not undergo apoptosis.

Not all patients respond similarly to treatments of malignant brain tumors, and individualized treatment for patients is often necessary. The induction of apoptosis in treatment-resistant CSC and survivin-positive cells may therefore be useful for the treatment of malignant tumors. Since CSC are in the G0 phase, they must be induced to differentiate or undergo apoptosis. The therapeutic outcomes may be further improved by investigating tumor resistance coupled with a combination of differentiation induction in resistant cells and increasing sensitivity to radiotherapy and chemotherapy. Methods of killing CSC have been investigated, such as knocking out the self-replicating ability through inhibiting molecules necessary for self-replication and development of an antibody preparation against cell surface antigens specifically expressed by CSC, such as anti-CD44 antibody against HER2 for breast cancer ${ }^{26)}$. The addition of therapies targeting treatment-resistant cells to the standard protocol may improve survival rates in patients with malignant brain tumors.

\section{Conflict of interest}

The authors have declared no conflict of interest.

\section{References}

1) Reifenberger G, Collins VP. Pathology and molecular genetics of astrocytic gliomas. J Mol Med. 2004;82:656-670.

2) Kleihues P, Burger PC, Aldape KD, et al. Glioblastoma. In Louis DN, Ohgaki H, Wiestler OD, et al. eds. WHO classification of tumours of the central nervous system. 4th ed. Lyon: International Agency for Research on Cancer; 2007. pp33-49.

3) Stoica G, Lungu G, Martini-Stoica H, et al. Identification of cancer stem cells in dog glioblastoma. Vet Pathol. 2009;46:391-406.

4) Hensley ML, Schuchter LM, Lindley C, et al. American Society of Clinical Oncology clinical practice guidelines for the use of chemotherapy and radiotherapy protectants. J Clin Oncol. 1999;17:3333-3355.

5) Walker MD, Alexander E Jr, Hunt WE, et al. Evaluation of BCNU and / or radiotherapy in the treatment of anaplastic gliomas. A cooperative clinical trial. J Neurosurg. 1978;49:333-343.

6) Kristiansen K, Hagen S, Kollevold T, et al. Combined modality therapy of operated astrocytomas grade III and IV. Confirmation of the value of postoperative irradiation and lack of potentiation of bleomycin on survival time: a 
prospective multicenter trial of the Scandinavian Glioblastoma Study Group. Cancer. 1981;47:649-652.

7) Bao $\mathrm{S}, \mathrm{Wu} \mathrm{Q}, \mathrm{McLendon} \mathrm{RE}$, et al. Glioma stem cells promote radioresistance by preferential activation of the DNA damage response. Nature. 2006;444:756-760.

8) Hide T, Takezaki T, Nakamura H, et al. Brain tumor stem cells as research and treatment targets. Brain Tumor Pathol. 2008;25:67-72.

9) Holland EC. Gliomagenesis: genetic alterations and mouse models. Nat Rev Genet. 2001;2:120-129.

10) Maher EA, Furnari FB, Bachoo RM, et al. Malignant glioma: genetics and biology of a grave matter. Genes Dev. 2001;15:1311-1333.

11) Brescia P, Ortensi B, Fornasari L, et al. CD133 is essential for glioblastoma stem cell maintenance. Stem Cells. 2013;31:857-869.

12) Cho DY, Lin SZ, Yang WK, et al. Targeting cancer stem cells for treatment of glioblastoma multiforme. Cell Transplant. 2013;22:731-739.

13) Beier D, Wischhusen J, Dietmaier W, et al. CD133 expression and cancer stem cells predict prognosis in highgrade oligodendroglial tumors. Brain Pathol. 2008;18:370-377.

14) Cheng L, Huang Z, Zhou W, et al. Glioblastoma stem cells generate vascular pericytes to support vessel function and tumor growth. Cell. 2013;153:139-152.

15) Li F, Ambrosini G, Chu EY, et al. Control of apoptosis and mitotic spindle checkpoint by survivin. Nature. 1998;396:580-584.

16) Ambrosini G, Adida C, Altieri DC. A novel anti-apoptosis gene, survivin, expressed in cancer and lymphoma. Nat Med. 1997;3:917-921.

17) Yagihashi A, Asanuma K, Nakamura M, et al. Detection of anti-survivin antibody in gastrointestinal cancer patients. Clin Chem. 2001;47:1729-1731.

18) Fields AC, Cotsonis G, Sexton D, et al. Survivin expression in hepatocellular carcinoma: correlation with proliferation, prognostic parameters, and outcome. Mod Pathol. 2004;17:1378-1385.

19) Chen JH, Chen KY, Ma HI, et al. Cortactin, fascin and survivin expression associated with clinicopathological parameters in brain gliosarcoma. Chin J Physiol. 2010;53:234-244.

20) Sasaki A, Udaka Y, Tsunoda Y, et al. Analysis of p53 and miRNA expression after irradiation of glioblastoma cell lines. Anticancer Res. 2012;32:4709-4713.

21) Bao $\mathrm{S}, \mathrm{Wu} \mathrm{Q}, \mathrm{McLendon} \mathrm{RE}$, et al. Glioma stem cells promote radioresistance by preferential activation of the DNA damage response. Nature. 2006;444:756-760.

22) Srinivasula SM, Ashwell JD. IAPs: what's in a name? Mol Cell. 2008;30:123-135.

23) Yamada Y, Kuroiwa T, Nakagawa T, et al. Transcriptional expression of survivin and its splice variants in brain tumors in humans. $J$ Neurosurg. 2003;99:738-745.

24) Mita AC, Mita MM, Nawrocki ST, et al. Survivin: key regulator of mitosis and apoptosis and novel target for cancer therapeutics. Clin Cancer Res. 2008;14:5000-5005.

25) Altieri DC. Survivin, cancer networks and pathway-directed drug discovery. Nat Rev Cancer. 2008;8:61-70.

26) Nadal R, Ortega FG, Salido M, et al. CD133 expression in circulating tumor cells from breast cancer patients: potential role in resistance to chemotherapy. Int J Cancer. 2013;133:2398-2407.

[Received June 20, 2013 : Accepted July 21, 2013] 